\title{
detasFLEX - A computational design tool for the analysis of various notch flexure hinges based on non-linear modeling
}

\author{
Stefan Henning, Sebastian Linß, and Lena Zentner \\ Compliant Systems Group, Technische Universität Ilmenau, Ilmenau, 98693, Germany \\ Correspondence: Stefan Henning (stefan.henning@tu-ilmenau.de)
}

Received: 10 August 2018 - Revised: 25 October 2018 - Accepted: 29 October 2018 - Published: 20 November 2018

\begin{abstract}
Notch flexure hinges are commonly used in compliant mechanisms for precision engineering applications and yet important rotational properties of a hinge like the bending stiffness, maximum angular deflection and rotational precision are difficult to predict accurately and simultaneously. There exist some closed-form equations and a few design tool approaches for calculating flexure hinges with particular geometries, but apart from that no comprehensive calculation program for the contour-specific analysis is known to the authors. Developed in MATLAB, this paper presents a novel computational design tool using a non-linear analytical approach for large deflections of rod-like structures to calculate the elasto-kinematic flexure hinge properties by numerically solving a system of differential equations. Building on previous investigations, four certain hinge contours are implemented, the circular, the corner-filleted, the elliptical, and the power function-based contour with different exponents. In addition to the theoretical approach and the implementation it is exemplarily shown, that finite elements method (FEM) results correlate well with the analytical design tool results. For a given deflection angle of $10^{\circ}$ and a corner-filleted contour as an example, the deviations of the bending stiffness are between $0.1 \%$ and $9.4 \%$ for typical parameter values. The presented design tool can be beneficial for the accelerated and systematic synthesis of compliant mechanisms with optimized flexure hinges.
\end{abstract}

\section{Introduction}

Notch flexure hinges experience a growing application in industry and research. Due to their advantages of high reproducibility, high resolution, and clearance-free or frictionless motion they are widely used in compliant mechanisms (Howell et al., 2013; Zentner, 2014) for precision engineering, micromechanics or measurement technology tasks. For these applications flexure hinges with specific notch geometries are the most common form of compliant segments used to realize a rotation (Lobontiu, 2003). According to bending of the materially coherent joint, the angular deflection is limited because of the resulting maximum strain. Further, a small shift of the axis of rotation results which affects the motion behavior as well as guidance accuracy of a compliant mechanism over its rigid-body counterpart (Venanzi et al., 2005).
Nevertheless, predicting the motion of an individual single-axis flexure hinge is a non-trivial task due to geometric non-linearities caused by large deflections, leading to a system of differential equations that need numeric solving approaches for accurately determining the hinge performance properties. When looking for an optimal flexure hinge design for a specific mechanism motion task predominantly timeconsuming simulations and iterative procedures become a necessity and only a few detailed guidelines are known to the authors. In literature closed-form equations exist for the prediction of the bending stiffness and deflection in most cases (e.g. Paros and Weisbord, 1965; Smith et al., 1987; Tseytlin, 2002; Wu and Zhou, 2002; Lobontiu, 2003; Schotborgh et al., 2005). Rarely, simple and contour-independent design equations for calculating various elasto-kinematic hinge properties are suggested (e.g. Linß et al., 2017a, b). Otherwise, when optimal hinge contours are looked for, design 
graphs may be used to figure out an appropriate order for polynomial flexure hinges ( $\operatorname{Lin} \beta, 2015)$.

To date specific tools for the analysis of flexure hinges are sparsely found. One author for example provides scripts that can be downloaded and used within MATHCAD to calculate flexure hinges and compliant mechanisms using simplified design-equations in a limited parameter range (Janssen, 2018). Other software-based applications for the analysis of circular notch flexure hinges are coming with a simple graphical user interface (Vink, 2018; van Beek, 2018). Additionally, inaccessible applications which are developed for published research are reported briefly (Ivanov, 2016). Most of these applications are based on the implementation of empirical design equations derived from analytical considerations or FEM analysis. When analyzing flexure hinges using these tools, the analyzing options are mostly very restricted. Most of these tools apply compromised design equations only within a small parameter range. Apart from that, more complex tools for the analysis (Megaro et al., 2017) and synthesis (Turkkan and Su, 2016; Culpepper and Kim, 2004) of compliant mechanisms with distributed compliance are state of the art. On the contrary they do neither offer the possibility to regard several notch flexure hinges nor calculate the rotational precision or axis shift. Moreover, it has to be mentioned that some of these software applications require licenses for commercial software like MATHCAD or MATLAB, but others are available for free. With this paper the authors try to fill the gap regarding a comprehensive software application for the accurate and non-linear analysis of various notch flexure hinges with the most important contours and broad evaluation criteria which may be advantageous especially for precision engineering to enable an intuitive and quick design process.

According to a plane rotation caused by bending due to a moment or transverse force load, this paper presents a novel design tool for the contour-specific quasi-static analysis of notch flexure hinges. The tool offers the calculation of parameters like bending stiffness, rotational axis shift, maximum elastic strain and outer fiber strain distribution, maximum angular deflection, and deformation of the neutral axis for extensive geometric specifications - computable for a specified load or rotational angle. The calculations will be possible by numerically solving a system of differential equations for large deflections of thin rod-like structures within a few seconds. Further, the design tool provides a plot of the hinge for the given geometry.

The following sections are organized as follows. In Sect. 2 the investigated types and regarded contours of flexure hinges are presented and characterized. In Sect. 3 the analytical approach for the characterization of flexure hinges with the theory of large deflections of rod-like structures based on non-linear modeling is described. Moreover, specific parameters to calculate the motion and strain are investigated, too. In Sect. 4 the development and implementation of the design tool are outlined. Also, the algorithm for the analyzing proce- dure is illustrated. In Sect. 5 an FEM-based characterization is done for four different hinge contours and results are compared to the design tool-based solution. Finally, conclusions are drawn in Sect. 6.

\section{Design and modeling of notch flexure hinges}

In the past numerous designs of flexure hinges with no limit to the geometric shape have been developed. It can mainly be differentiated between cut-out geometries and more complex compositions of the compliant segment. An example for a complex geometry would be the proposed butterfly hinge (e.g. Henein et al., 2003; Pei and Yu, 2011) or a topology optimization-based contour (Zhu et al., 2014). However, in this paper the focus is on notch flexure hinges with distinct contour shapes. For this purpose, many notch geometries are being outlined in literature. By far the most commonly used and easy to manufacture circular flexure hinge contour (e.g. Paros and Weisbord, 1965; Wu and Zhou, 2002) is well known for its large bending stiffness and high precision but also for high maximum strain values. On the contrary, a well distributed strain can be achieved with the corner-filleted contour (e.g. Lobontiu, 2003; Meng et al., 2013). As a compromise between high precision and low strain the elliptical contour has been applied (e.g. Smith et al., 1997; Chen et al., 2008). Moreover, parabolic or hyperbolic (e.g. Lobontiu, 2003; Chen et al., 2009) and cycloidal contours (Tian et al., 2010) have been utilized as well as combinations of the mentioned basic geometries (e.g. Zelenika et al., 2009; Lobontiu et al., 2011; Chen et al., 2011).

Beyond that, more complex mathematical functions like the spline contour (Christen and Pfefferkorn, 1998; De Bona and Munteanu, 2005), the power function-based contour ( $\mathrm{Li}$ et al., 2013) the exponent-sine contour (Wang et al., 2013) the Lamé contour (Desrochers, 2008) and the Bézier contour (Vallance et al., 2008) are rarely taken into consideration. Lately higher order polynomial functions were proposed to regulate the bending stiffness, precision and elastic strain distribution to an optimum (Linß et al., 2011a, 2015; Gräser, et al., 2018). For combining the advantages of different notch contours, asymmetric flexure hinges were previously considered (Chen et al., 2005). Also the kinematic behavior in terms of an ideal axis of rotation can be realized with asymmetric flexure hinges (Linß et al., 2011b; Lin et al., 2013). However, in this paper only transversal and axial symmetric notch flexure hinges are investigated because they allow a holistic and intuitive design with regard to the mechanism synthesis. The investigated design and modeling of notch flexure hinges are described in the following subsections.

\subsection{Investigated flexure hinges and geometric parameters}

Subject of this research is a notch flexure hinge fixed at one end and loaded at the free end as it is shown in Fig. 1 for the 


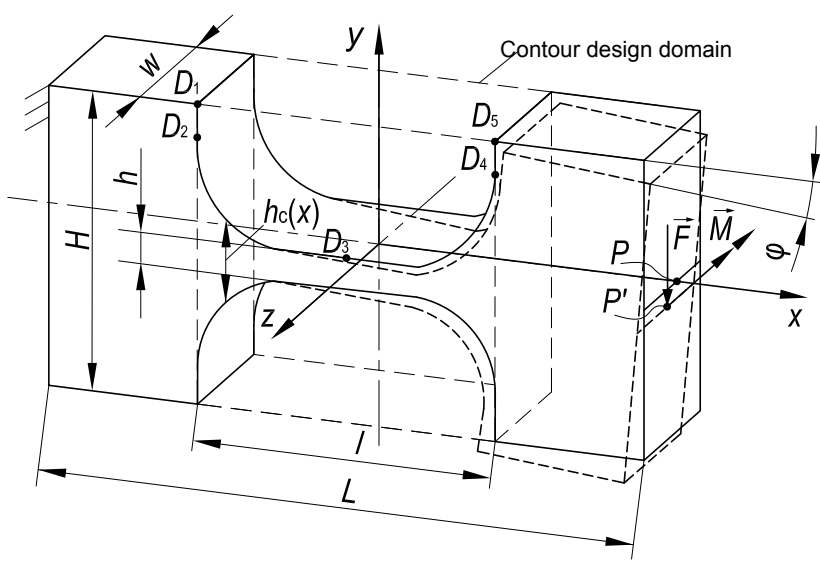

Figure 1. Notch flexure hinge with a variable hinge height within the contour design domain, the geometric parameters and the deflected state as a result of a moment and/or a transverse force load.

initial and the deflected state. With respect to the application in a mechanism and according to literature, the flexure hinge with its notch contour design domain is generally modeled with adjacent link segments (Yong et al., 2008). The deflection can be modeled in two ways by either specifying the load (a moment load $M$, a transverse force load $F$, or a moment and force load $M, F$ ) or by specifying the deflection angle $\varphi$ at the free end in point $P$. The illustration of the flexure hinge in Fig. 1 shows the basic geometric parameters that define the shape of the hinge: the total hinge height $H$, minimum hinge height $h$, hinge width $w$, total length $L$, contour length $l$, and contour function $h_{\mathrm{c}}(x)$ based on the selected notch geometry.

In this paper only directionally constant forces are accounted and the equations are based on them. The system of differential equations will vary for follower forces and may be adjusted for those applications. The vectorial direction for a given moment is $\boldsymbol{M}=-\boldsymbol{M} \boldsymbol{e}_{z}$, for a transverse force $\boldsymbol{F}=-F \boldsymbol{e}_{y}$ and for the bending angle $\boldsymbol{\varphi}=-\varphi \boldsymbol{e}_{z}$. For characterizing different flexure hinges, the dimensionless ratios in Table 1 are introduced with their lower and upper bounds in the software application. These lower and upper bounds are chosen to aim for that the dimensions of the cross sections are small compared to the hinge length and therefore to support the validity of the implemented theory incorporating rod-like structures.

\subsection{Flexure hinge contour selection and definition}

Regarding Fig. 1, the design domain for the variable notch contour ranges from point $D_{1}$ to $D_{5}$ along the $x$-axis while the contour will always be axial-symmetric to the $y$-axis. Due to the used theory for all investigations it must be assured that the hinge contour is a symmetric, continuously differentiable, and not undercut contour function. The hinge has a rectangular cross-section, and the minimum notch height $h$ is at the origin of the hinge (at $x=0$ ). In some occasions, the
Table 1. Geometric flexure hinge parameters related to the total hinge height and their parameter ranges.

\begin{tabular}{lcrr}
\hline Parameter & Ratio & $\begin{array}{r}\text { Lower } \\
\text { bound }\end{array}$ & $\begin{array}{r}\text { Upper } \\
\text { bound }\end{array}$ \\
\hline Hinge overall length & $\beta_{L}=\frac{L}{H}$ & $\beta_{l}$ & 10 \\
Contour length & $\beta_{l}=\frac{l}{H}$ & 0.1 & $\beta_{L}$ \\
Minimum contour height & $\beta_{h}=\frac{h}{H}$ & 0.01 & 1 \\
Hinge width & $\beta_{w}=\frac{w}{H}$ & 0.1 & 100 \\
\hline
\end{tabular}

contour will contain a straight line parallel to the $y$-axis in between $D_{1}$ and $D_{2}$ and analogically in between $D_{4}$ and $D_{5}$, and therefore the resulting curvature will include a discontinuity. With this regard, the integration when solving will be performed ranging from the points $D_{2}$ to $D_{4}$ to ensure a continuously differentiable mathematical expression. This can be done by adjusting the initial value of the curvature for each segment when a discontinuity is present.

In the design tool three typical flexure hinge contours are considered, the circular (Fig. 2a), corner-filleted (Fig. 2b) and elliptical contour (Fig. 2c). Besides that, the advantages of the polynomial contour ( $\operatorname{Lin} ß$ et al., 2011a) are implemented and extended to a power function (Fig. 2d) to offer a wider range of possible contours. The power function-based hinge contour offers a great spectrum for the flexure hinge design ( $\operatorname{Lin} \beta, 2015)$. Due to the variable height function, the contour design may be adjusted from circular-type to corner-filletedtype contours to achieve a desired angular deflection or axis shift.

In Table 2 the according height functions of the four implemented flexure hinge contours are listed. Contour-specific parameters for the exact definition of each flexure hinge are proposed with $R, r, r_{x}, r_{y}, n$ as they are shown in Fig. 2 . The circular and elliptical flexure hinge contours may be exact or approximated semi-circles or semi-ellipses depending on the chosen geometry. This issue is depicted in Fig. 3 for the circular hinge contour and in Fig. 4 for the elliptical contour. The circular hinge contour only describes an exact semicircle for $2 R \leq H-h$ (cf. Fig. 3a and b). The notch length in these two cases results as:

$l=2 R$.

Otherwise the circle center is positioned outside of the contour design domain (cf. Fig. 1) and approximated semicircles result (cf. Fig. 3c) with the notch length expressed as:

$l=2 \sqrt{R^{2}-\left(\frac{H-h}{2}-R\right)^{2}}$.

The same accounts for the elliptical contour. An exact semiellipse can only be modeled for $2 r_{y} \leq H-h$ (cf. Fig. 4a 


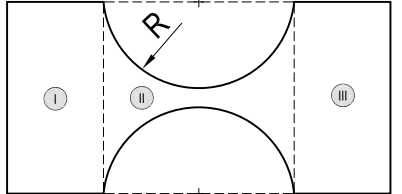

(a)

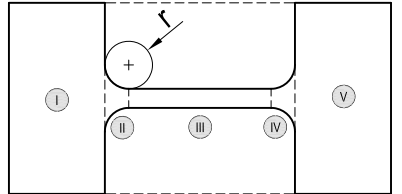

(b)

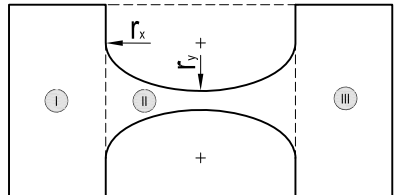

(c)

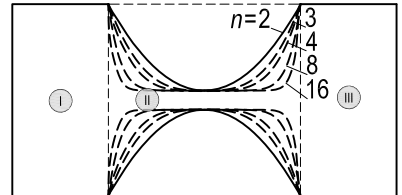

(d)

Figure 2. The four implemented flexure hinges with their contour-specific parameters: (a) circular contour with radius $R$, (b) corner-filleted contour with stress-optimal fillet radius $r=0.1 l$ (Linß et al., 2011b), (c) elliptical contour with major axis $r_{x}$ and minor axis $r_{y}$, (d) variable power function-based contour (shown for different exponent values $n$ ).

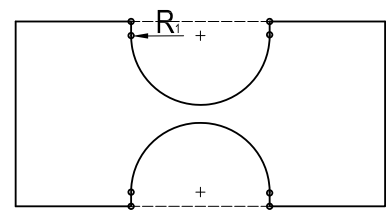

(a)

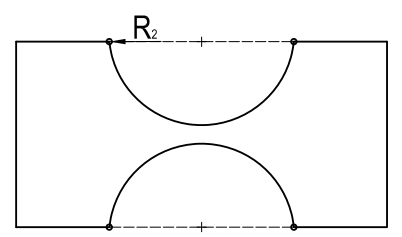

(b)

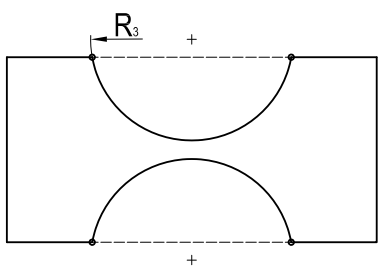

(c)

Figure 3. Three different cases for designing a circular flexure hinge in dependence of the input parameter values (all shown for $\beta_{L}=2$ and $\beta_{h}=0.1$ ): (a) $2 R<H-h$ (with $\beta_{l}<1$ ), (b) $2 R=H-h$ (right semi-circular contour, with $\beta_{l}<1$ ), (c) $2 R>H-h$ (with $0<\beta_{l}<\beta_{L}$ ).

and $b)$ and the notch length is as follows:

$l=2 r_{x}$.

In case the ellipse center is located outside of the contour design domain, approximated semi-ellipses arise with the notch length as:

$l=2 r_{x} \sqrt{1-\left(\frac{H-h}{2 r_{y}}-1\right)^{2}}$.

\section{Theoretical approach}

In contrast to form and force-closed joints the materially coherent connection of flexure hinges leads to a restoring force when bent (bending stiffness) - which can be advantageous in technical systems, too. Apart from that, the angular deflection of a flexure hinge is limited by reaching admissible material stress or strain values (maximum angular deflection). Moreover, no exact relative rotation is possible with a flexure hinge because always a shift of its axis of rotation occurs in dependence of geometric and load parameters (rotational precision). When applying flexure hinges in a compliant mechanism, this can lead to path deviations compared to the rigid-body mechanism, which are not negligible especially in precision engineering (Venanzi et al., 2005; Linß, 2015).

In this section, the approach of the non-linear analytical characterization of a notch flexure hinge and the equations for its bending stiffness, strain distribution/maximum angular elastic deflection, and rotational precision are presented.

\subsection{Theory for large deflections of rod-like structures}

As long as the dimensions of a cross section are small compared to the rod length, the non-linear theory for large deflections of rod-like structures is sufficient to describe the motion behavior of compliant systems (Zentner, 2014). If a flexure hinge is modeled together with adjacent deformable link segments as a bent beam with a variable height, this theory is assumed to be suitable for the calculations in this paper, too. Therefore, the assumption is made that Bernoulli's hypothesis, Saint-Venant's principle, and Hooke's law apply. Shear deformation according to Timoshenko as investigated in (Dirksen and Lammering, 2011) and the effect of anticlastic bending (Campanile et al., 2011) are neglected, because first investigations with the applied theory show a good correlation between the theoretical results and FEM simulations for different flexure hinges (Linß et al., 2017b).

A stationary coordinate system $\xi \eta \zeta$ is considered with the origin at the fixed end (Fig. 5). Bending of a beam results in a deflection curve of the neutral axis with assumed constant length. Therefore, the arc length parameter $s$ is introduced to describe the neutral axis in its deflected state. The rod undergoes a shift $u_{\xi}(s)$ and $u_{\eta}(s)$ for each point along $s$. Because of the shift in $\xi$ and $\eta$-direction a deflection angle $\theta(s)$ results. The resulting curvature is defined by $\kappa(s)$ as the gradient of the deflection angle $\theta(s)$. Thus, for describing a flexure hinge as a bent rod or beam four non-linear differential equations result:

$\frac{\mathrm{d} M_{\zeta}}{\mathrm{d} s}+F \cos \theta=0$ 


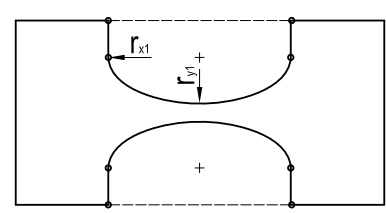

(a)

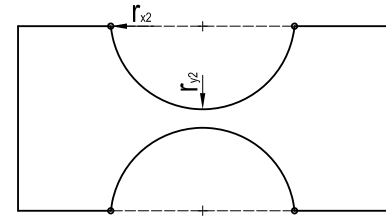

(b)

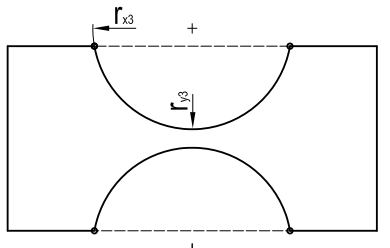

(c)

Figure 4. Three different cases for designing an elliptical flexure hinge in dependence of the input parameter values (all shown for $\beta_{L}=2$, $\beta_{h}=0.1$ with $0<\beta_{l}<\beta_{L}$ ): (a) $2 r_{y}<H-h$, (b) $2 r_{y}=H-h$ (right semi-elliptical contour), (c) $2 r_{y}>H-h$.

Table 2. Height functions of the four implemented flexure hinge contours.

\begin{tabular}{ll}
\hline Hinge contour & Height function expression \\
\hline Circular & $h_{\mathrm{c}}(x)=h+2 R-2 \sqrt{R^{2}-x^{2}}$ \\
Corner-filleted & $h_{\mathrm{c}}(x)= \begin{cases}h+2 r-2 \sqrt{r^{2}-\left(x+\frac{l}{2}-r\right)^{2}}, & \text { for }-\frac{l}{2} \leq x<-\frac{l}{2}+r \\
h, & \text { for }-\frac{l}{2}+r \leq x \leq \frac{l}{2}-r \\
h+2 r-2 \sqrt{r^{2}-\left(x-\frac{l}{2}+r\right)^{2}}, & \text { for } \frac{l}{2}-r<x \leq \frac{l}{2}\end{cases}$ \\
Elliptical & $h_{\mathrm{c}}(x)=h+2 r_{y}\left(1-\sqrt{\left.1-\frac{x^{2}}{r_{x}^{2}}\right)}\right.$ \\
Power function & $h_{\mathrm{c}}(x)=h+\frac{(H-h)}{\left(\frac{l}{2}\right)^{n}|x|^{n} ; \text { with } n \in \mathbb{R} \text { and } 1.1 \leq n \leq 50}$ \\
\hline
\end{tabular}

$\frac{\mathrm{d} \theta}{\mathrm{d} s}-\kappa=$, with $\kappa=\frac{M_{\zeta}}{E I_{\zeta}}$ and

$I_{\zeta}=\left\{\begin{array}{ll}\frac{w H^{3}}{12} & \text { for } 0 \leq s<L_{1}-\frac{l}{2} \\ \frac{w h_{\mathrm{c}}^{3}(s)}{12} & \text { for } L_{1}-\frac{l}{2} \leq s \leq L_{1}+\frac{l}{2} \\ \frac{w H^{3}}{12} & \text { for } L_{1}+\frac{l}{2}<s \leq L_{1}+L_{2}\end{array}\right.$,

$\frac{\mathrm{d} u \xi}{\mathrm{d} s}-\cos \theta+1=0$

$\frac{\mathrm{d} u_{\eta}}{\mathrm{d} s}-\sin \theta=0$

Since the flexure hinge is symmetric, no initial curvature of the beam is regarded in this paper. Due to the fact that simple and concise closed-form equations cannot be derived from this system of non-linear differential equations a numerical solution is done with the following boundary conditions for a moment load at the free end of the hinge:

$\kappa(L)=\frac{M}{E I_{\zeta}(L)}, \theta(0)=0, u_{\xi}(0)=0, u_{\eta}(0)=0$,

and for a transverse force load with:

$\kappa(L)=0, \theta(0)=0, u_{\xi}(0)=0, u_{\eta}(0)=0$.
The boundary value problem is solved numerically with the use of MATLAB as it is described in Sect. 4.2. At the end of this procedure all four parameters $\kappa, \theta, u_{\xi}$ and $u_{\eta}$ are obtained for each point $s$ along the deformed neutral axis and further results can be determined as it is described in the following subsections. Please note, though the equations have been derived for a universal approach based on a positive deflection, the input force, moment or deflection angle are translated into negative values by the software. This way the flexure hinge is always deflected into $\eta$ and $\xi$-direction like depicted in Fig. 5. Nevertheless, because of the symmetry, it makes no difference in which direction the hinge is deflected. Therefore, it is focused on the absolute value of the angular deflection.

\subsection{Bending stiffness of a flexure hinge}

On the contrary to the compliance of a flexure hinge, the bending stiffness around an axis of a system of coordinates is a measure for the resistance of an object against deformation under external loads. Due to the fact that flexure hinges in this paper are mainly exposed to bending around the $\zeta$ axis, the stiffness is described as a product of the Young's modulus and the geometrical moment of inertia. Therefore, it becomes clear that the bending stiffness can be influenced 


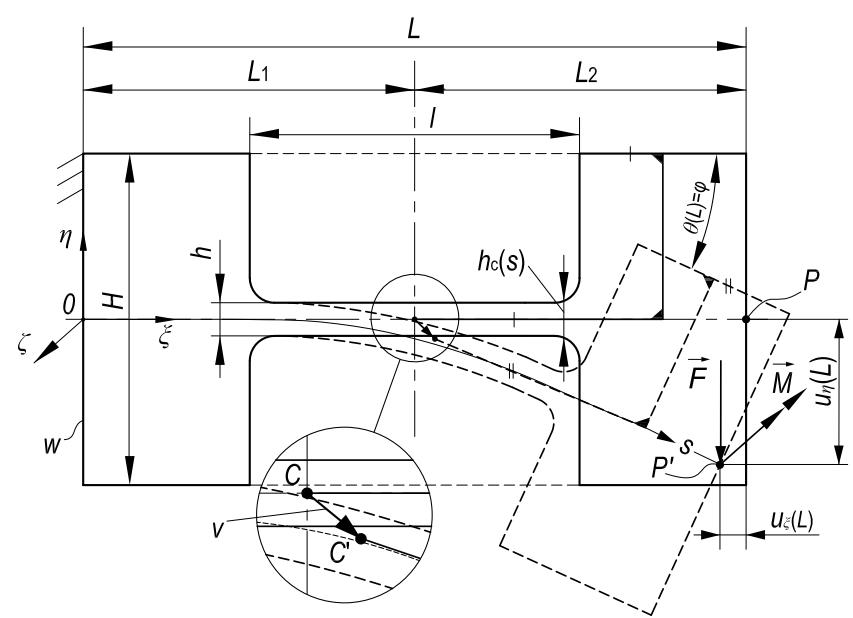

Figure 5. Parameters for the theoretical characterization of a flexure hinge (depiction of the initial and deflected position) with the model for the determination of the rotational axis shift based on guiding the center with a constant distance, the fixed center approach.

by material and geometric parameter changes. When speaking about bending stiffness properties in this paper, depending on the load case the relation between the load and the rotation angle, $M(\varphi)$ and $F(\varphi)$, are meant.

\subsection{Strain distribution and maximum angular deflection of a flexure hinge}

The bending stress $\sigma$ is analyzed after linear beam theory to characterize the maximum stress of the entire flexure hinge for a given deflection angle as a result of the moment or force load:

$\left.\sigma(s)\right|_{\eta_{\max }}=-\frac{M_{\zeta}(s)}{I_{\zeta}(s)} \cdot \eta_{\max }(s)$.

According to the used theory, the maximum absolute stress value always results at the outer fiber for the maximum coordinate of $\eta$, which corresponds with the contour height function of the flexure hinge. Fulfilling Hooke's law, stress and strain are linearly connected by the Young's modulus as it is described for the bending strain $\varepsilon$ as:

$\varepsilon(s)=\frac{\sigma(s)}{E}=-\frac{M_{\zeta}(s)}{E I_{\zeta}(s)} \cdot \eta_{\max }(s)=-\kappa(s) \cdot \eta_{\max }(s)$.

Further, a part of Eq. (12) can be expressed with the curvature $\kappa(s)$. Due to the solution of the system of differential equations in MATLAB the curvature along the neutral axis is known and can therefore be used to determine the elastic strain. This solution is obtained for each specification and load case of the analysis settings. The maximum elastic strain can then be found with an accuracy that depends on the step size of the solution for $\kappa(s)$. According to the approach, the maximum strain is independent from the width $w$ for a given deflection. Due to the used theory, only the bending moment $M_{\zeta}$ is taken into account.

For a moment load, the maximum strain occurs, related to the $\xi$-axis in the hinge center in general. In contrast to this, for a force load the critical location depends on the flexure hinge contour. For this purpose, the strain distribution can be plotted along the entire flexure hinge so that a user can assess critical strain areas. The maximum absolute value of the strain $\varepsilon_{\max }$ is implemented in the results window of the design tool. Among the four regarded contours, the circular contour always leads to the highest strain values for a constant deflection (see Sect. 5). Using the elliptical, the power function-based, and the corner-filleted contour, the maximum strain value can be further reduced and analyzed with the software for different material choices. The admissible elastic strain $\varepsilon_{\text {adm }}$ should always be higher than the maximum strain of each flexure. A safety factor is introduced to emphasize the relation between admissible and maximum elastic strain according to Eq. (13):

$S_{\mathrm{F}}=\frac{\varepsilon_{\mathrm{max}}}{\varepsilon_{\mathrm{adm}}}$

The user should always aim for a safety factor $S_{\mathrm{F}}>1$ to ensure that no plastic deformation arises. Using the calculated safety factor, the maximum angular deflection can be expressed by Eq. (14):

$\varphi_{\max }=S_{\mathrm{F}} \cdot \varphi$

Therein $\varphi$ will either be the user-defined deflection angle or the calculated deflection angle in case a load is given. This way the contour and geometry-dependent correlation between the material-given admissible elastic strain $\varepsilon_{\mathrm{adm}}$ and the maximum rotation angle $\varphi_{\max }$ is derived.

\subsection{Rotational precision of a flexure hinge}

Due to the fact that flexure hinges do not have a stationary rotation axis, the term rotational precision is introduced. Notably, in precision engineering, the rotational precision of a flexure hinge is a very important performance criterion. Because of the serial connection of several flexure hinges in the kinematic chain of a compliant mechanism, the rotational axis shift $v$ of a single flexure hinge (cf. Fig. 5) can influence the path deviation of a coupler point of the compliant mechanism compared to the rigid-body mechanism. Depending on the possibility to specify the deflection angle or load case, $v(\varphi)$ or $v(M, F)$ is considered in this paper. In literature several approaches are suggested to express the rotational precision which lead to slightly different results. Most approaches are based on the deflected state of the hinge so that the shift of the center point at $\xi=\frac{L}{2}$ is calculated (e.g. Lobontiu, 2003; Tian et al., 2010). Another approach considers the intersection of the $\xi$-axis and the tangent to the deflected neutral axis 
through $P^{\prime}$. This way the axis shift can be determined in $\xi$ direction only (e.g. Horie et al., 1997; Smith, 2000). Further, the fixed centrode is calculated with the help of a geometrical approximation method (e.g. Dirksen and Lammering, 2011; Palmieri et al., 2012), which leads to high numerical effort because of a theoretically infinitesimal analysis. As it has been described in previous publications (e.g. Tseytlin, 2002; Zelenika et al., 2009; Linß et al., 2011b), the distance between the initial center point $C$ and the guided center point $C^{\prime}$ with a constant distance of $L_{2}$ during the motion (fixed center approach) is suitable and chosen to define the rotational precision $v$ in this paper (cf. Fig. 5).

The absolute value of the rotational axis shift $v$, based on the fixed center approach, is put together from the axis shift in $\xi$ and $\eta$-direction as:

$$
\begin{aligned}
v & =\sqrt{\left(\xi_{C^{\prime}}-L_{1}\right)^{2}+\eta_{C^{\prime}}^{2}} \\
& =\sqrt{\left(L_{2}+u_{\xi}-L_{2} \cos \varphi\right)^{2}+\left(u_{\eta}-L_{2} \sin \varphi\right)^{2}} .
\end{aligned}
$$

To determine the axis shift according to Eq. (15), the coordinates $\xi$ and $\eta$ at the free end of the hinge on the neutral axis $s$ and the rotation angle $\varphi$ must be known. The numerical solution of the system of differential equations provides results for all $s$ in both axes shifts so the coordinates of point $P^{\prime}$ are known, too. The deflection angle $\varphi$ will therefore equal the angular deflection in point $P^{\prime}$ which is derived from the differential equation of $\theta(s)$ using $\varphi=\theta(L)$.

Furthermore, to minimize the axis shift, the user should know, that independent from the hinge contour a transverse force leads to a significant larger axis shift than a moment load for the same angle $\varphi$. Also the hinge contour has a strong influence on the axis shift, depending on the dimensions $\beta_{l}$ and especially $\beta_{h}$ (e.g. Linß et al., 2017a). With regard to a high rotational precision or a small axis shift the following order can be generalized for thin hinges: The circular contour, the elliptical contour or polynomial 4th-order contour, and the corner-filleted contour. It can be concluded that the more the compliance is distributed along the hinge contour (meaning longer thin stretches), the higher the axis shift will be. On the contrary, a more concentrated compliance leads to a better rotational precision.

\section{Implementation}

In this section the development of a computer program for the analysis of notch flexure hinges with different hinge contours will be described. For this purpose, the previously described theory for large deflections of rod-like structures will be implemented in a graphical user interface (GUI) developed with MATLAB. Therefore, a solution for the non-linear analytical characterization will be received and evaluated. In the following subsections it is shown how the different inputs and selections are realized throughout the GUI (front end) and how they are internally processed by the software (back end) to produce the results which are then displayed in fields and diagrams. Further it is explained how the software will be distributed for license-free public usage. For the development of the GUI the MATLAB version R2017b was used.

\subsection{Matlab-based graphical user interface}

The developed GUI for the analysis of notch flexure hinges is shown in Fig. 6. For public usage it was generated as a standalone application called "detasFLEX" using the MATLAB deploy tool for WINDOWS 64 bit computers. Due to this process, no MATLAB license is needed to run the program. Although, the file comes with a Runtime - a database including all the important MATLAB functions - that needs to be downloaded and installed to the computer.

The development of the GUI has been made with the use of the GUI development environment, called GUIDE. It basically is a layout editor where one can graphically design the appearance of an application using input and output text fields, push buttons, sliders, axes and more. GUIDE automatically generates the MATLAB code for modifying the program behavior. This way it is possible to process input data, solve the system of differential equations and generate all result data and diagrams.

\subsection{Program structure}

For a better overall understanding for a user to analyze a specific flexure hinge using the given design tool, the modular composition of the program is shown in Fig. 7 which can be derived from the GUI in Fig. 6. Mainly the interface is divided into four groups. To start with, there are text fields for the input of data and information about the hinge notation and valid geometries for the chosen contour. In this area the material, the hinge contour, the dimensional and the contourspecific geometric parameters of the flexure hinge may be set. Next the analysis settings can be made. Thereby the program user can choose between the selection of a given deflection angle or a given load and specify its value, set the load case and finally press the "calculate"-button or reset everything to default values. Afterwards, when all results are obtained by the solver, the result values are presented in a separate section of the interface. Also, the geometry, bending line, bending stiffness and precision as well as the strain distribution are charted in diagrams. In addition, features to export data and diagrams into files, print the geometry and zoom into the notation are provided.

Internally an algorithm based on the user input and analysis settings is executed to compute a solution for the boundary value problem and supply all the important results and diagrams. The underlying procedure is showcased in the form of a flowchart in Fig. 8 and will be briefly described. When first executing the program "detasFLEX.exe" a user may specify input values for material and basic geometry, choose 


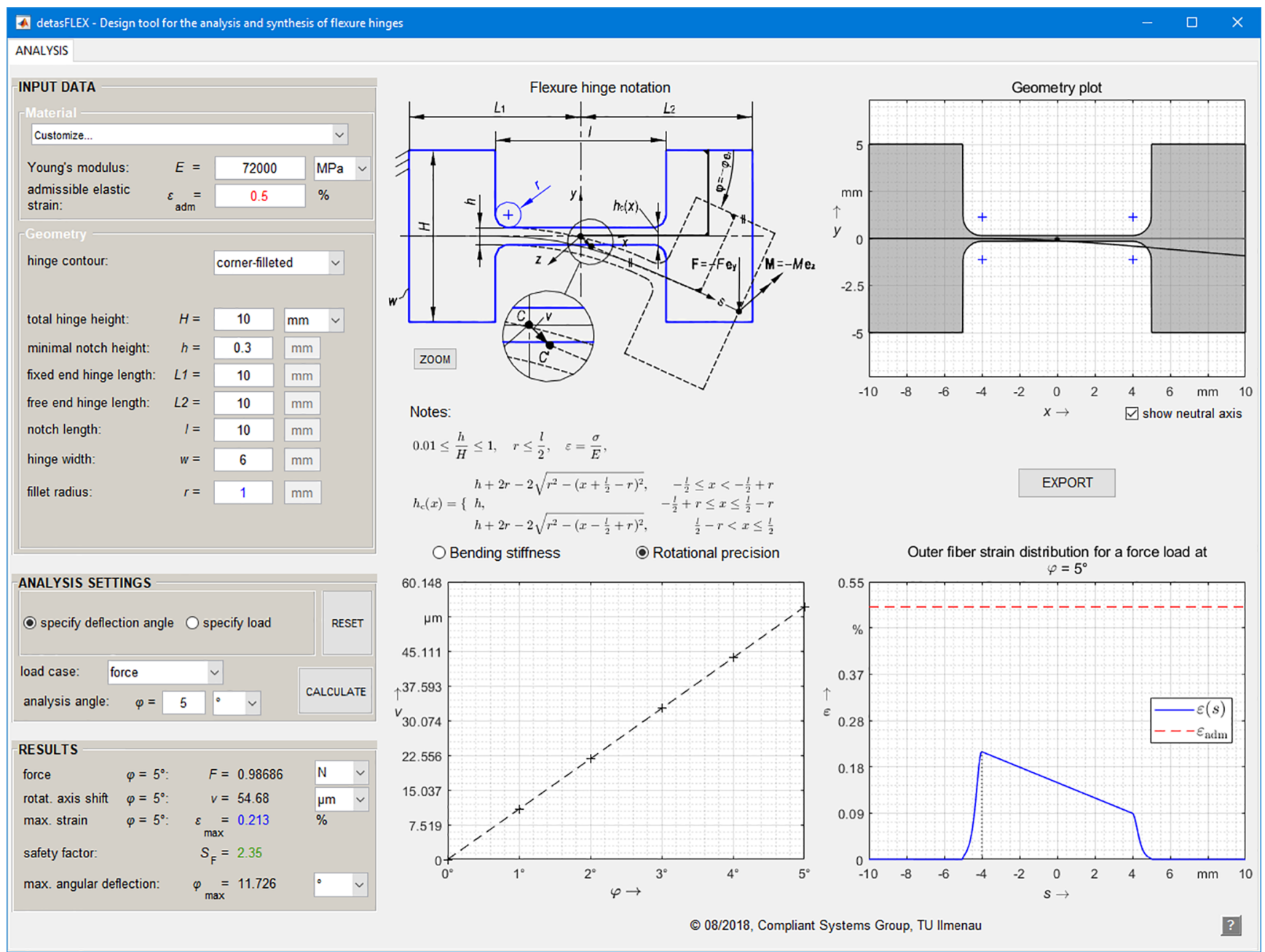

Figure 6. Graphical user interface of the PC program detasFLEX (design tool for the analysis of flexure hinges), shown using the example of a corner-filleted flexure hinge.

a contour and manipulate contour specific parameters. These values and selections get read and converted into SI units for further processing. To make sure all input data comply with the geometric bounds and are numeric real positive data greater zero, a query is programmed. In the case of invalid inputs, a warning will be displayed and parameters need to be adjusted by the user. Following, a constant step size along $-L_{1} \leq x \leq L_{2}$ is being set as $t_{\mathrm{c}}=L \times 10^{-3}$ for the calculation and plotting of the flexure hinge geometry. Afterwards, the program determines if either the deflection angle or the load at the free end of the flexure hinge is specified which reflects major differences between the solution.

In case a deflection angle is specified, firstly the angle value is divided into five load steps. It has been shown that the motion behavior for very large angular deflections may accurately be calculated with the used theory (Zentner et al., 2017) which is why the angle was limited to $\varphi \leq 45^{\circ}$. Thereafter, the chosen load case (moment or force) needs to be evaluated. Next, initial values are estimated. In a following step, the initial guess is approximated for a cantilever beam, fixed at $\xi=0$ with the constant height $h$ and a deflection of $\varphi(L)=1^{\circ}$ because the extent of the deflection mainly depends on the minimum notch height and so the solver does not exaggerate the deflection on the first adjustment step. The initial guesses are derived from linear beam theory for small deflections and result for a moment load as

$M_{\mathrm{ini}}=1^{\circ} \cdot \frac{\pi}{180^{\circ}} \cdot \frac{E w h^{3}}{12\left(L_{1}+L_{2}\right)}$,

and for a force load results as

$F_{\text {ini }}=1^{\circ} \cdot \frac{\pi}{180^{\circ}} \cdot \frac{E w h^{3}}{6\left(L_{1}+L_{2}\right)^{2}}$.

In case the load is specified, the program will first figure out which load case (moment, force or both) was set by the user and then split the load into five equally sized load steps. Then, for each load step the system of differential equations 


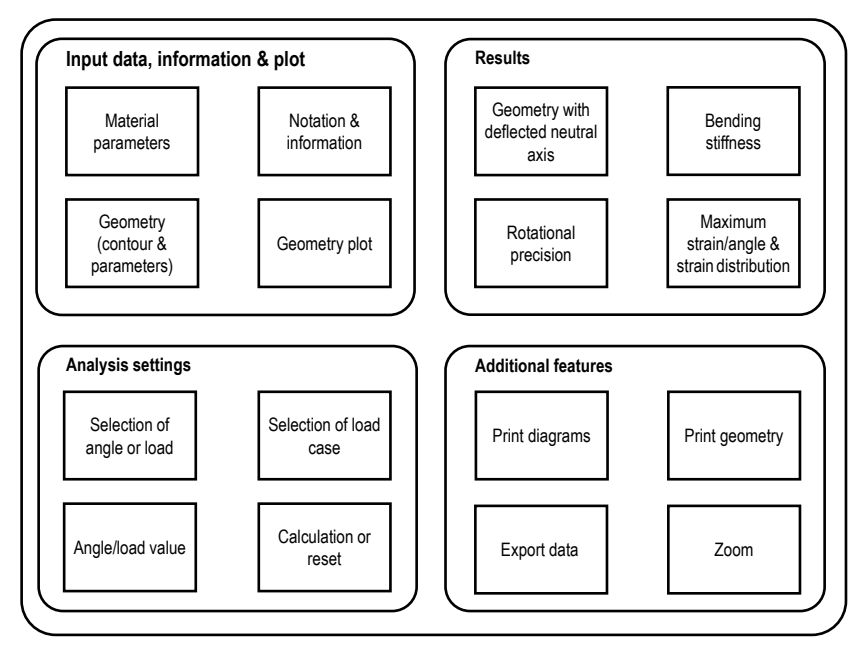

Figure 7. Modular structure of the PC program detasFLEX (Analysis).

gets solved with the used MATLAB function ode45 with a variable step size $t_{\mathrm{v}}$ for efficient calculation. The solver is based on the Dormand-Prince method which uses six function evaluations in each step. It represents an explicit fourstep Runge-Kutta method. The algorithm performs an iterative integration of first order non-stiff differential equations with initial conditions. Afterwards a solution is obtained for the system of differential equations. In case of a force load, a boundary value problem is present because of the unknown support reaction due to the $\xi$-shift of the free end (important for large deflections). For this purpose, the function $f$ solve (shooting method for the unknown moment support reaction called $M_{A \zeta}$ ) is applied to iterate the solution until the boundary conditions are matched. In case of a moment load no optimization needs to be done because all support reactions are explicit. It can be taken advantage of the linear relation $M(\varphi)$ so that the system of differential equations only needs to be solved numerically for $M_{\text {ini }}$ (using ode45) and with the resulting angle $\theta(L)=\varphi$ the related moment $M(\varphi)$ may be obtained. Resulting values of the results $M$ or $F, \varphi, \kappa, \theta$, $u_{\xi}$ and $u_{\eta}$ are then being saved for further processing. It can be mentioned, that the elapsing time for the numerical solution is usually shorter for plain moment loads because of the simplifications possible.

In a following step, when a solution for the system of differential equations was found, the deflected state of the neutral axis is plotted into the diagram. Also the bending stiffness and axis shift are evaluated with the given equations described in Sect. 3 and outlined in their respective chart. Next, the outer fiber strain distribution and maximum value are being calculated with the step size $t_{\mathrm{v}}$ and also displayed in a graph. The safety factor and all other output values are printed into the intended results window. The whole process takes just a few seconds after the "calculate" button is pressed.

\subsection{Segment-wise calculation}

As it is already shown in Fig. 2, the flexure hinges are split up into several sections for the numerical solution. Beam sections with a constant hinge height are solved by referring to a constant geometrical moment of inertia whilst sections containing a variable contour function are referred to the solution of the corresponding height function found in Table 2. The system of differential equations therefore varies for each section. The circular, the elliptical and the power functionbased contour are split up into three sections (cf. Fig. 2a, c and d) and the corner-filleted contour is split up into five separate sections when being calculated (cf. Fig. 2b). The numerical solution at the end of each section serves as a set of initial values for the next section. This method guarantees a continuous progression of the neutral axis. Furthermore, using this approach, transition points arise at the exact location in between sections along the neutral axis which enable the evaluation of results precisely at these points. This is especially important when it comes to examining the rotational precision of the regarded flexure hinge.

\subsection{Examples}

To demonstrate some of the functionalities the design tool offers, examples are given in this subsection. The program enables a wide variety of different geometry, material, contour and analysis settings selections so that numerous notch flexure hinges for diverse tasks in industry and research may be analyzed within a few seconds with respect to the geometric lower and upper bounds.

The following examples are done for all four different contours with the same typical geometric ratios $\left(\beta_{L}=2, \beta_{l}=\right.$ $\left.1, \beta_{h}=0.03, \beta_{w}=0.6\right)$ and material settings $(E=72 \mathrm{GPa}$, $\varepsilon_{\mathrm{adm}}=0.5 \%$ ). Firstly a specific given deflection angle of $\varphi=5^{\circ}$ is defined and each hinge is then calculated for a force load. The obtained geometry, deflection curve and strain distribution diagrams are presented in Fig. 9. It becomes clear that the deflection angle is too high for the chosen circular and elliptical flexure hinge contour because the admissible elastic strain is exceeded. Further it is noticeable, that due to the load case of a force load, the maximum elastic strain is shifted out of the hinge center towards the fixed end of the hinge, the more distributed the compliance of the hinge is. This is especially significant for the power function-based contour (Fig. 9c) and even more for the corner-filleted contour (Fig. 9d). Also, the more distributed the compliance is along the hinge contour (larger sections with a small hinge height), the lower the maximum strain will be which is well visible for the corner-filleted contour. Also the bending line is more equally curved throughout the whole length $L$, the more distributed the compliance of the hinge is. From Fig. 9a to $d$ the compliance of the hinge increases and so does the outer fiber strain distribution. 


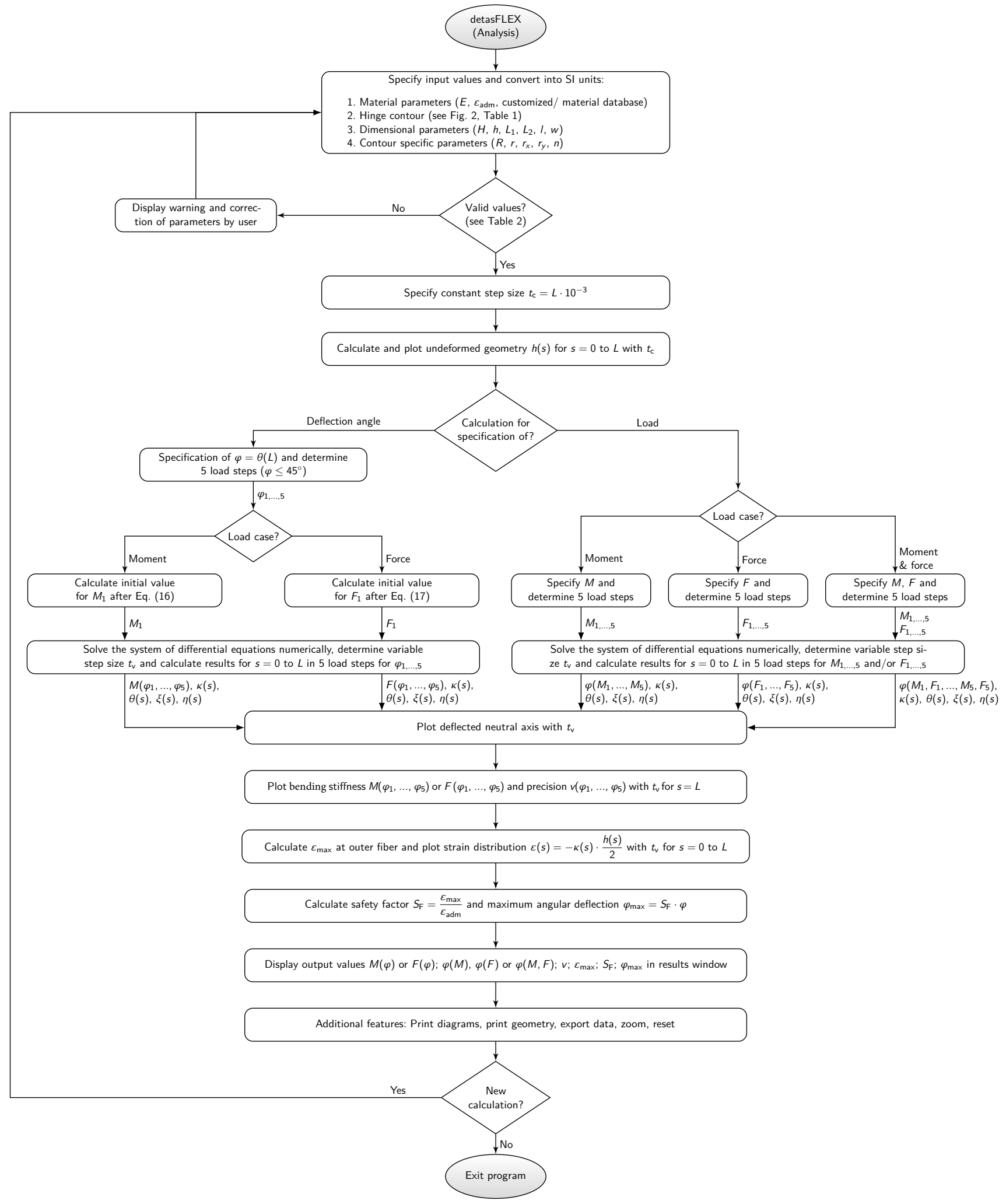

Figure 8. Detailed flowchart of the PC program detasFLEX. 


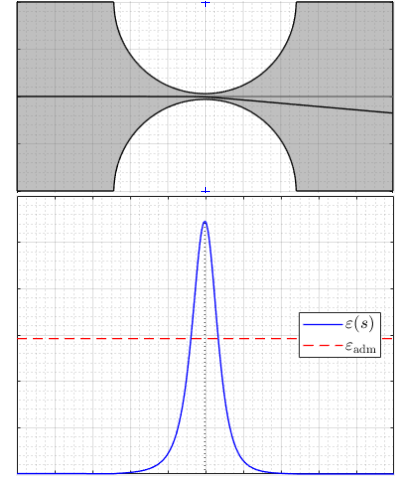

(a)

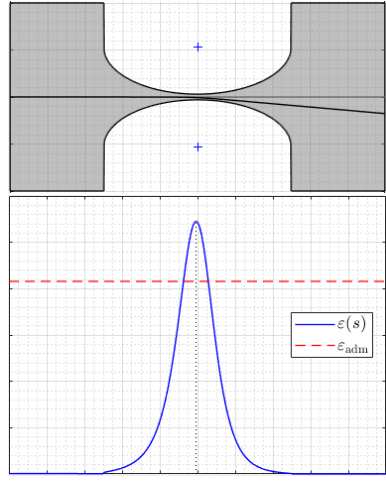

(b)

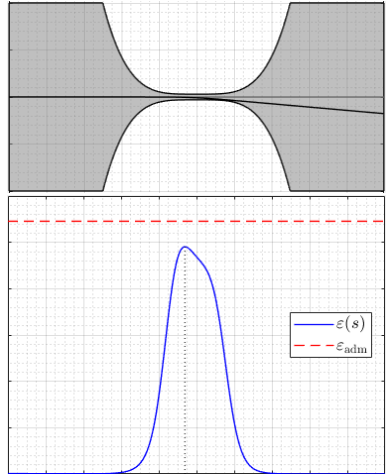

(c)

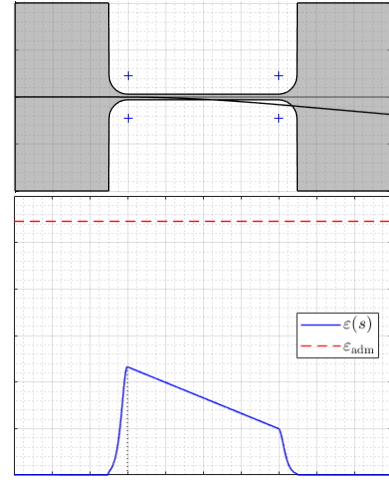

(d)

Figure 9. Example results of the deformed neutral axis and the outer fiber strain distribution for a force load computed for an angle input of $|\varphi|=5^{\circ}\left(\beta_{L}=2, \beta_{l}=1, \beta_{h}=0.03\right.$; result values see Table 3): (a) circular contour, (b) elliptical contour, (c) power function-based contour with $n=4$, (d) corner-filleted contour.

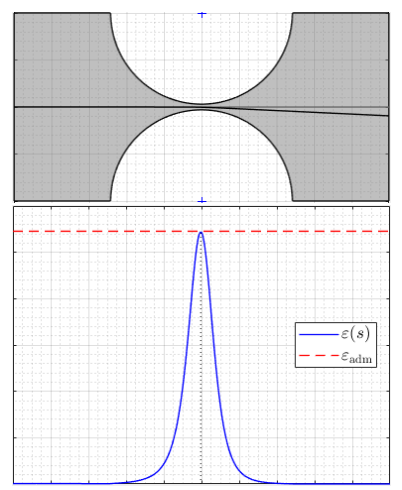

(a)

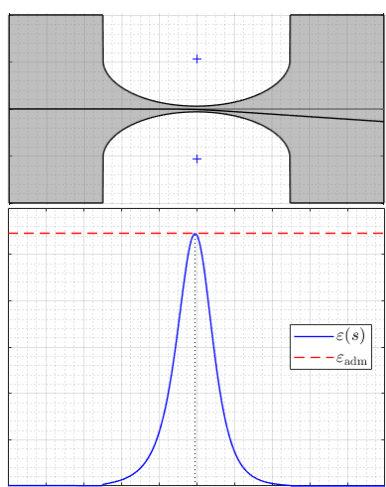

(b)

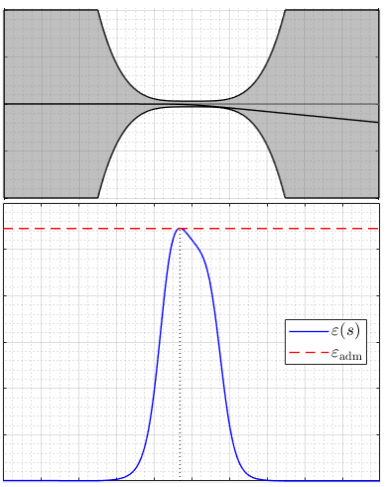

(c)

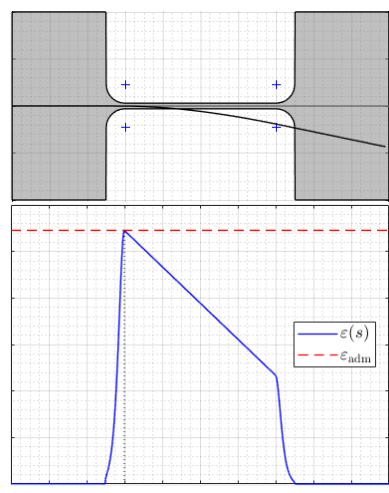

(d)

Figure 10. Example results of the deformed neutral axis and the outer fiber strain distribution for different force load inputs to equal $\varepsilon_{\mathrm{adm}}=0.5 \%\left(\beta_{L}=2, \beta_{l}=1, \beta_{h}=0.03\right)$ : (a) circular contour with $\left|\varphi_{\max }\right|=2.72^{\circ}$, (b) elliptical contour with $\left|\varphi_{\max }\right|=3.81^{\circ},(\mathbf{c})$ power function-based contour with $n=4$ and $\left|\varphi_{\max }\right|=5.56^{\circ}$, (d) corner-filleted contour with $\left|\varphi_{\max }\right|=11.73^{\circ}$.

In another case study, which is presented in Fig. 10, the same four flexure hinges are regarded but with different analysis settings. For this application example, the maximum angular deflection that is derived from the previous example is taken as an input for $\varphi$. The maximum angular deflection varies for each hinge in accordance to the compliance distribution. The circular flexure hinge (Fig. 10a) offers the lowest possible angular deflection in this case with $\varphi_{\max }=2.72^{\circ}$ due to the highest bending stiffness compared to the cornerfilleted contour (Fig. 10d) with $\varphi_{\max }=11.73^{\circ}$. Nevertheless, the circular flexure hinge realizes the lowest shift of the axis of rotation. When applying $\varphi_{\max }$, the maximum elastic strain equals the admissible elastic strain as it is visible in the strain distribution diagrams in Fig. 10. According to the non-linear theory the deformation of the neutral axis in $\xi$-direction is obvious, especially for the corner-filleted flexure hinge (cf. Sect. 2). Design tool users may adjust the hinge contour, the dimensional or contour-specific parameters to influence the maximum possible angular deflection, the maximum elastic strain or the rotational stiffness and precision to suit the flexure hinge design to their needs.

\section{FEM-based verification}

To confirm the analytical design tool-based results and therefore the usability of the provided PC program, finite elements method is considered to compare and verify the implementation of the non-linear deformation theory. For the FEM-based 3-D structural simulation ANSYS Workbench 18.2 was used. The CAD model and FEM model are shown in Fig. 11. For the determination of the rotational precision in FEM, the same approach as it is described in Sect. 3.4 is considered by adding an additional part onto the CAD model according to the chosen fixed center approach (cf. Fig. 5) to measure the distance between $C$ and $C^{\prime}$ (Fig. 11a). The FEM model is considered with a fixed support on one side and it is free on 
Table 3. FEM-based verification and comparison of the contour-specific and load-dependent results for a discrete angular deflection of $|\varphi|=5^{\circ}\left(\beta_{L}=2, \beta_{l}=1, \beta_{h}=0.03, \beta_{w}=0.6\right)$ with specification of the relative deviations to the analytical design tool solution.

\begin{tabular}{|c|c|c|c|c|c|c|c|c|c|}
\hline \multirow[t]{2}{*}{ Hinge contour } & \multirow[t]{2}{*}{ Method } & \multicolumn{4}{|c|}{ Moment load } & \multicolumn{4}{|c|}{ Force load } \\
\hline & & $M[\mathrm{Nm}]$ & $v[\mu \mathrm{m}]$ & $\varepsilon_{\max }[\%]$ & $\left|\varphi_{\max }\right|\left[^{\circ}\right]$ & $F[\mathrm{~N}]$ & $v[\mu \mathrm{m}]$ & $\varepsilon_{\max }[\%]$ & $\left|\varphi_{\max }\right|\left[^{\circ}\right]$ \\
\hline \multicolumn{10}{|c|}{ Circular with $R=5 \mathrm{~mm}$} \\
\hline & analytical & 0.0592 & 1.411 & 0.914 & 2.736 & 5.944 & 4.358 & 0.919 & 2.720 \\
\hline & FEM & 0.0622 & 1.428 & 0.892 & 2.803 & 6.240 & 5.020 & 0.894 & 2.796 \\
\hline & deviation & $5.1 \%$ & $1.2 \%$ & $2.4 \%$ & $2.4 \%$ & $5.0 \%$ & $15.2 \%$ & $2.7 \%$ & $2.8 \%$ \\
\hline \multicolumn{10}{|c|}{ Corner-filleted with $r=0.1 l$} \\
\hline & analytical & 0.0098 & 5.485 & 0.151 & 16.534 & 0.987 & 54.675 & 0.213 & 11.727 \\
\hline & FEM & 0.0102 & 5.367 & 0.166 & 15.060 & 1.025 & 54.720 & 0.228 & 10.965 \\
\hline & deviation & $4.1 \%$ & $2.2 \%$ & $9.9 \%$ & $8.9 \%$ & $3.9 \%$ & $0.1 \%$ & $7.0 \%$ & $6.5 \%$ \\
\hline \multicolumn{10}{|c|}{ Elliptical, with $r_{x}=2 r_{y}=0.5 l$} \\
\hline & analytical & 0.0420 & 1.943 & 0.649 & 3.854 & 4.235 & 7.843 & 0.656 & 3.811 \\
\hline & FEM & 0.0446 & 1.906 & 0.641 & 3.900 & 4.481 & 8.551 & 0.639 & 3.912 \\
\hline & deviation & $6.2 \%$ & $1.9 \%$ & $1.2 \%$ & $1.2 \%$ & $5.8 \%$ & $9.0 \%$ & $2.6 \%$ & $2.7 \%$ \\
\hline \multicolumn{10}{|c|}{ Power function with $n=1.5$} \\
\hline & analytical & 0.1614 & 0.741 & 2.490 & 1.004 & 16.076 & 1.660 & 2.481 & 1.008 \\
\hline & FEM & 0.1557 & 0.825 & 2.476 & 1.010 & 15.632 & 2.310 & 2.478 & 1.009 \\
\hline & deviation & $3.5 \%$ & $11.3 \%$ & $0.6 \%$ & $0.6 \%$ & $2.8 \%$ & $39.2 \%$ & $0.1 \%$ & $0.1 \%$ \\
\hline \multicolumn{10}{|c|}{ Power function with $n=2.0$} \\
\hline & analytical & 0.0818 & 1.042 & 1.263 & 1.979 & 8.217 & 2.585 & 1.269 & 1.970 \\
\hline & FEM & 0.0852 & 1.138 & 1.368 & 1.827 & 8.555 & 3.140 & 1.369 & 1.826 \\
\hline & deviation & $4.2 \%$ & $9.2 \%$ & $8.3 \%$ & $7.7 \%$ & $4.1 \%$ & $21.5 \%$ & $7.9 \%$ & $7.3 \%$ \\
\hline \multicolumn{10}{|c|}{ Power function with $n=3.0$} \\
\hline & analytical & 0.0402 & 1.680 & 0.620 & 4.029 & 4.038 & 5.650 & 0.634 & 3.946 \\
\hline & FEM & 0.0427 & 1.758 & 0.694 & 3.602 & 4.285 & 6.242 & 0.694 & 3.602 \\
\hline & deviation & $6.2 \%$ & $4.6 \%$ & $11.9 \%$ & $10.6 \%$ & $6.1 \%$ & $10.5 \%$ & $9.5 \%$ & $8.7 \%$ \\
\hline \multicolumn{10}{|c|}{ Power function with $n=4.0$} \\
\hline & analytical & 0.0278 & 2.226 & 0.428 & 5.839 & 2.785 & 9.459 & 0.450 & 5.562 \\
\hline & FEM & 0.0294 & 2.190 & 0.414 & 6.039 & 2.946 & 9.980 & 0.439 & 5.695 \\
\hline & deviation & $5.9 \%$ & $1.6 \%$ & $3.3 \%$ & $3.4 \%$ & $5.8 \%$ & $5.5 \%$ & $2.4 \%$ & $2.4 \%$ \\
\hline \multicolumn{10}{|c|}{ Power function with $n=8.0$} \\
\hline & analytical & 0.0156 & 3.599 & 0.240 & 10.412 & 1.561 & 23.792 & 0.279 & 8.954 \\
\hline & FEM & 0.0164 & 3.651 & 0.270 & 9.259 & 1.643 & 24.180 & 0.298 & 8.389 \\
\hline & deviation & $5.4 \%$ & $1.4 \%$ & $12.5 \%$ & $11.1 \%$ & $5.2 \%$ & $1.6 \%$ & $6.8 \%$ & $6.3 \%$ \\
\hline \multicolumn{10}{|c|}{ Power function with $n=16.0$} \\
\hline & analytical & 0.0115 & 4.718 & 0.178 & 14.050 & 1.157 & 40.568 & 0.228 & 10.963 \\
\hline & FEM & 0.0119 & 4.731 & 0.198 & 12.658 & 1.207 & 40.738 & 0.236 & 10.593 \\
\hline & deviation & $3.2 \%$ & $0.3 \%$ & $11.0 \%$ & $9.9 \%$ & $4.3 \%$ & $0.4 \%$ & $3.5 \%$ & $3.4 \%$ \\
\hline
\end{tabular}

the opposite side, while the free end is loaded with a moment or a directionally constant transverse force load (Fig. 11b). The evaluation of two points on the free end guarantee an accurate approximation of the deflection angle $\varphi$. Therefore, the relations $M(\varphi)$ and $F(\varphi)$ may be outlined. Further, the maximum angular deflection $\varphi_{\max }$ can be determined in dependence of the analyzed maximum equivalent elastic strain.

According to literature, flexure hinges with very variable dimensions are generally modeled as a 3-D solid structure (Zettl et al., 2005) and if possible with adjacent link segments (Yong et al., 2008) in all FEM simulations in this paper. The latter accounts for the considerations for the analytical characterization in Sect. 3, too. Moreover, large deflections are also considered in the FEM analysis settings for an accurate comparison with the analytical calculations due to the non-linear beam theory. Other assumptions are a linear material behavior and a comparable and fine discretization of the hinge for all the different contours. The mesh of the FEM model is chosen to be finely divided in areas of the notch and especially in areas of the minimum hinge height $h$ (Fig. 11b).

In an example FEM analysis for typical geometric parameter values $\left(\beta_{L}=2, \beta_{l}=1, \beta_{h}=0.03, \beta_{w}=0.6\right)$ and all four 


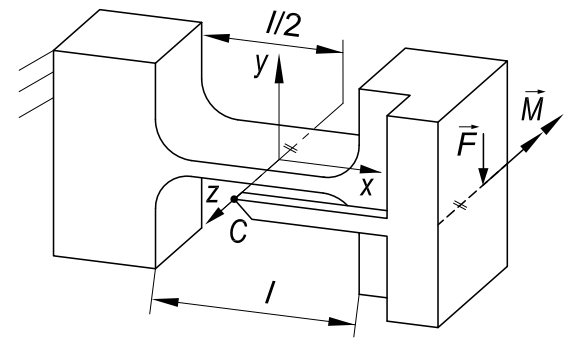

(a)

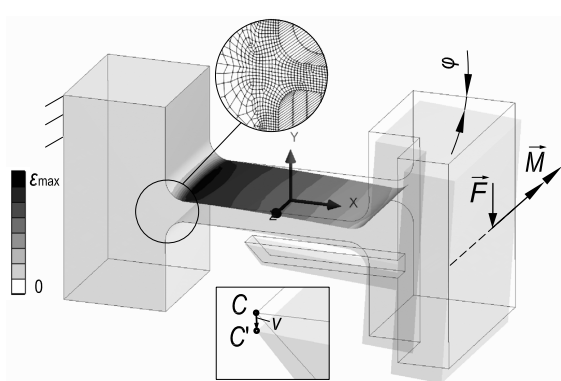

(b)

Figure 11. FEM-based characterization of a flexure hinge: (a) CAD model, (b) FEM model with deformed hinge and mesh details.

hinge contours, the moment or force load is iterated until the deflection angle equals exactly $\varphi=5^{\circ}$ (Table 3 ) or $\varphi=10^{\circ}$ (Fig. 11b) for all investigations. Other than that, an aluminum alloy AW 7075 is chosen as a material which has been used for multiple high-precision engineering applications and surveys before (e.g. Gräser et al., 2017). The power functionbased contour is investigated for different exponent values $n$. In total, nine different flexure hinge contours are regarded and compared using both approaches in Table 3 . The bending stiffness, rotational precision, maximum elastic strain and the maximum rotational angle are mentioned for a moment and a force load at the free end of each hinge. In addition, the percentage deviation of the FEM results to the analytical design tool results is indicated, too.

Generally, the results are in good correlation with one another. Regarding the bending stiffness, the maximum deviation of the FEM results compared to the analytical results is $6.2 \%$ which is indicated to be very precise. Regarding the three criteria load $(M$ or $F), \varepsilon_{\max }$ and $\varphi_{\max }$, the errors slightly increase, while these higher errors occur for the maximum elastic strain. This is based on the fact, that in the analytical approach only the bending moment $M_{\zeta}$ is taken into consideration while in the FEM analysis other effects are taken into account, too. For example, within the finite-elements calculations shear deformation is taken into account which is neglected in the analytical approach. Likewise, especially in dependence of the flexure hinge width, minimum height and curvature radius, non-linear anticlastic bending (Campanile et al., 2011) may result which is not considered in the used theory. Furthermore, the von-Mises criterion is considered for the FEM-based maximum strain values. Nevertheless, the results do correlate well with deviations of only $12.5 \%$ at maximum.

In particular, greater differences may be found in terms of the rotational precision, especially when the hinge is deflected with a force load. With reference to the rotational precision it becomes clear that smaller absolute values for the axis shift in the micrometer range generally lead to higher deviations between FEM and analytical approaches. For example very small values of approximately $v=2 \mu \mathrm{m}$, in case of a power function-based contour with $n=1.5$ for a force load, lead to a deviation of $39.2 \%$. In contrast to that, for a corner-filleted contour with the same dimensions the deviation is only $0.1 \%$ whilst the absolute value is approximately $v=54 \mu \mathrm{m}$.

Another reason for these differences may be the fact that in the analytical approach no elongation of the neutral axis by tensile forces is considered, whereas they are possible in the FEM analysis. Because these strains are of the same magnitude as the axis shift, the discrepancy may be explained. In addition to that, the results in Table 3 provide insight in a correlation between the necessary load for achieving a deflection angle of $\varphi=5^{\circ}$ and the deviation of the rotation precision between the FEM result and the analytical solution. The more concentrated the compliance along the hinge contour is designed, e.g. for the circular contour or the power function-based contour with $n=1.5$, the higher the bending stiffness and the more load is necessary to deflect the hinge with $\varphi=5^{\circ}$. In the case of higher necessary loads, the tensile forces in FEM increase and so do the deviations to the analytical solution in terms of the rotational axis shift because of the higher elongation of the neutral axis in FEM.

Another investigation is exemplarily done for cornerfilleted hinges with regard to the bending stiffness for a force load and different total hinge length ratios $\beta_{L}=$ $\{1,2,10,20\}$, hinge width ratios $\beta_{w}=\{0.1,1\}$ and minimum hinge height ratios $\beta_{h}=[0.03,0.1]$ to illustrate the percentage deviation between FEM and analytical design tool solution for a larger parameter value range (Fig. 12). Though the design tool enables calculations for deflection angles of up to $45^{\circ}$, the presented stiffness deviations have been derived for an angular deflection of $10^{\circ}$, due to the fact that these deflections are typically sufficient in precision engineering applications. The investigation of the error of the force load $\Delta F$ shows a strong dependence of the dimensional parameters $\beta_{L}, \beta_{w}$ and $\beta_{h}$. For example it becomes obvious that very narrow flexure hinges $\left(\beta_{w}=0.1\right)$ generally lead to lower deviations and that the deviation increases with $\beta_{h}$ in this case. This is due to the fact, that for low minimum hinge height ratios the theory, which requires small dimensions of the cross sections compared to the rod length, is fulfilled more. With regard to the influence of the overall length $\beta_{L}$ it needs 


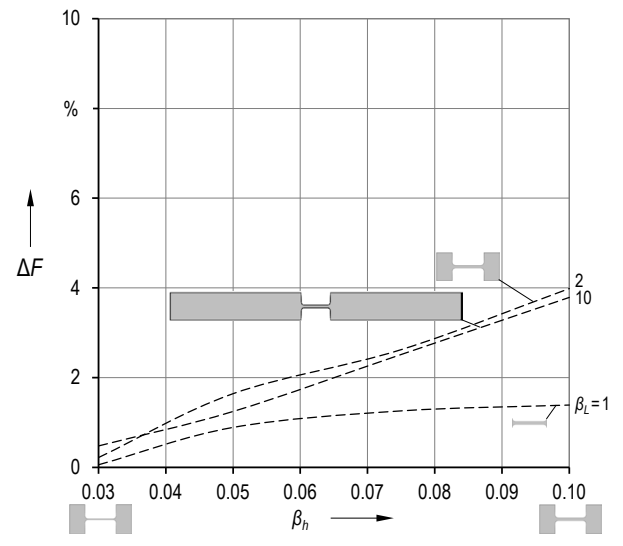

(a)

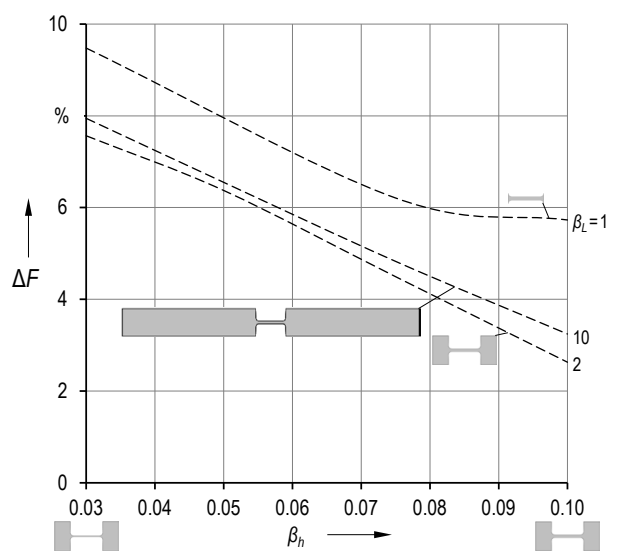

(b)

Figure 12. Parameter study of a corner-filleted flexure hinge with $\beta_{l}=1$ under the parameter variations $\beta_{L}=\left\{1,2,10\right.$ and $\beta_{h}=[0.03,0.1]$ showing the force deviation $\Delta F$ of the FEM results compared to the design tool results computed for an angle input of $|\varphi|=10^{\circ}:\left(\right.$ a) $\beta_{w}=$ 0.1 , (b) $\beta_{w}=1$.

to be noted that against the general recommendations (cf. Sect. 2) for small values very short adjacent links $\left(\beta_{L}=1\right)$ are present. In this case, the requirements of the theory of rod-like structures are again met more accurately.

Overall, the stiffness errors are in a range of $0.1 \%$ and $9.4 \%$. Thus, the non-linear analytical approach used within the design tool may be accounted appropriate for the implemented typical parameter value ranges. It may be expected to receive larger deviations between FEM and the analytical method for deflection angles larger than $10^{\circ}$, because the increasing tensile forces on the neutral axis are not considered in the developed program. Nevertheless, it is possible to receive a first estimate of the flexure hinge deformation and motion using the given design tool for large angular deflections, too.

\section{Conclusions}

In this paper a non-linear analytical approach for modeling various notch flexure hinges realizing an in-plane rotation is considered and implemented in the form of a design tool using the theory for large deflections of rod-like structures. The design tool was developed with MATLAB as a standalone software application which only requires the licensefree Runtime environment. Four different certain hinge contours are chosen and implemented in the design tool, the circular, corner-filleted, elliptical and power function-based contour. Various geometric and material parameters may be realized to allow for a broad usability in different cases. The analysis is possible for a moment and a transverse force load case as well as both loads combined for different lengths of both hinge sides. All three cases may be computed with a given load or deflection angle up to $45^{\circ}$. A variety of output parameters are outlined and the main hinge performance properties like the deformed neutral axis, the bending stiff- ness, the rotational precision and the elastic strain distribution are illustrated in the form of diagrams. Furthermore, a preview of the hinge geometry and instant visualization of input changes is realized in the GUI. Also, values for the angle or load, axis shift, strain distribution, maximum strain and maximum possible angle are obtained. Depending on the contour the bending stiffness deviation between FEM results and the design tool results is in the range of $0.1 \%$ and $9.4 \%$ for a deflection angle of $10^{\circ}$ and a corner-filleted contour. The results of both approaches coincide well. Thus, the program enables a wide variety of different geometry, material, and contour selections as well as multiple analysis criteria and settings so that numerous notch flexure hinges for diverse tasks not only in precision engineering industry and research may be accurately analyzed within a few seconds with respect to the geometric restrictions.

The presented design tool contributes to an accelerated contour-specific quasi-static analysis of the elasto-kinematic properties of notch flexure hinges with no need for iterative and time-consuming simulations. It therefore may be used for the systematic angle-dependent synthesis of compliant mechanisms with differently optimized flexure hinges (Linß et al., 2015; Gräser et al., 2018). Further research may earmark a synthesis part in the design tool so that required hinge properties may be predefined and an appropriate hinge contour with its suitable geometric parameter values will be proposed by the design tool. Other considerations like shear deformation, which make the program results more accurate, especially for specific cases of non-typical geometries, may be implemented, too. The influence of geometric scaling which is important for precision applications may also be considered (Linß et al., 2018). Additional features like the import of existing arbitrary geometries are possible. 
Data availability. The design tool can be requested for free on the following website: https://www.tu-ilmenau.de/nsys/download/ detasflex (Henning and Linß, 2018).

Author contributions. SH and SL developed the software detasFLEX and wrote the paper. SL did the FEM-based verification, reviewed the results and the paper. LZ supported the implementation of the theory for large deflections of rod-like structures.

Competing interests. The authors declare that they have no conflict of interest.

Acknowledgements. The authors would like to gratefully acknowledge the support of the German Research Foundation (DFG) under Grant No. ZE 714/10-2.

Edited by: Marek Wojtyra

Reviewed by: two anonymous referees

\section{References}

Campanile, L. F., Jähne, R., and Hasse, A.: Exact analysis of the bending of wide beams by a modified elastica approach, Proc. Inst. Mech. Eng. C, 11, 2759-2764, https://doi.org/10.1177/0954406211417753, 2011.

Chen, G., Jia, J.-Y., and Li, Z.-W.: Right-circular corner-filleted flexure hinges, IEEE International Conference on Automation Science and Engineering, Edmonton, Canada, 249-253, https://doi.org/10.1109/COASE.2005.1506777, 2005.

Chen, G., Shao, X., and Huang, X.: A new generalized model for elliptical arc flexure hinges, Rev. Sci. Instrum., 79, 95103, https://doi.org/10.1063/1.2976756, 2008.

Chen, G., Liu, X., Gao, H., and Jia, J.: A generalized model for conic flexure hinges, Rev. Sci. Instrum., 80, 55106, https://doi.org/10.1063/1.3137074, 2009.

Chen, G., Liu, X., and Du, Y.: Elliptical-Arc-Fillet Flexure Hinges: Toward a Generalized Model for Commonly Used Flexure Hinges, J. Mech. Des., 133, 81002, https://doi.org/10.1115/1.4004441, 2011.

Christen, G. and Pfefferkorn, H.: Nachgiebige Mechanismen: Aufbau, Gestaltung, Dimensionierung und experimentelle Untersuchung, VDI-Berichte Nr. 1423, VDI-Getriebetagung, Kassel, Germany, 309-329, 1998.

Culpepper, M. L. and Kim, S.: A Framework and Design Sythesis Tool Used to Generate, Evaluate and Optimize Compliant Mechanism Concepts for Research and Education Activities, ASME 2004 International Design Engineering Technical Conferences \& Computers and Information in Engineering Conference, Salt Lake City, Utah, 1583-1588, https://doi.org/10.1115/DETC2004-57606, 2004.

De Bona, F. and Munteanu, M. G.: Optimized Flexural Hinges for Compliant Micromechanisms, Analog Integr. Circ S., 44, 163174, https://doi.org/10.1007/s10470-005-2597-7, 2005.

Desrochers, S.: Optimum design of simplical uniaxial accelerometers, master thesis, McGill University, Montréal, 2008.
Dirksen, F. and Lammering, R.: On mechanical properties of planar flexure hinges of compliant mechanisms, Mech. Sci., 2, 109-117, https://doi.org/10.5194/ms-2-109-2011, 2011.

Gräser, P., Linß, S., Zentner, L., and Theska, R.: Design and Experimental Characterization of a Flexure Hinge-Based Parallel Four-Bar Mechanism for Precision Guides, Microactuators and Micromechanisms, Mechanisms and Machine Science, 45, Springer, Cham, 139-152, https://doi.org/10.1007/9783-319-45387-3_13, 2017.

Gräser, P., Linß, S., Zentner, L., and Theska, R.: Optimization of Compliant Mechanisms by Use of Different Polynomial Flexure Hinge Contours, in: 3rd IAK, Interdisciplinary Applications of Kinematics, Lima, Peru, 5-7 March 2018.

Henein, S., Spanoudakis, P., Droz, S., Myklebust, L. I., and Onillon, E.: Flexure pivot for aerospace mechanisms, 10th European Space Mechanisms and Tribology Symposium, San Sebastian, Spain, 2003.

Henning, S. and Linß, S.: detasFLEX - Design tool for the analysis and synthesis of flexure hinges, available at: https://www. tu-ilmenau.de/nsys/download/detasflex, last access: 14 November 2018.

Horie, M., Nozaki, T., Ikegami, K., and Kobayashi, F.: Design System of Superelastic Hinges and Its Application to Micromanipulators, JSME Int. J., 40, 323-328, https://doi.org/10.1299/jsmec.40.323, 1997.

Howell, L. L., Magleby, S. P., and Olsen, B. M.: Handbook of Compliant Mechanisms, Wiley, Chichester, 2013.

Ivanov, I.: Methodical Development of a Parallel Kinematic Positioning System Based on Monolithic Structures with Flexure Hinges, doctoral thesis, RWTH Aachen, Aachen, https://doi.org/10.18154/RWTH-2016-11304, 2016.

Janssen, H.: Flexure hinge or elastic hinge, available at: https://www.janssenprecisionengineering.com/page/ flexure-hinge-or-elastic-hinge, last access: 23 July 2018.

Li, Q., Pan, C., and Xu, X.: Closed-form compliance equations for power-function-shaped flexure hinge based on unit-load method, Precis. Eng., 37, 135-145, https://doi.org/10.1016/j.precisioneng.2012.07.010, 2013.

Lin, R., Zhang, X., Long, X., and Fatikow, S.: Hybrid flexure hinges, Rev. Sci. Instrum., 84, 085004, https://doi.org/10.1063/1.4818522, 2013.

Linß, S.: Ein Beitrag zur geometrischen Gestaltung und Optimierung prismatischer Festkörpergelenke in nachgiebigen Koppelmechanismen, doctoral thesis, TU Ilmenau, Ilmenau, nbnresolving.de/urn:nbn:de:gbv:ilm1-2015000283, 2015.

Linß, S., Erbe, T., and Zentner, L.: On polynomial flexure hinges for increased deflection and an approach for simplified manufacturing, $13^{\text {th }}$ World Congress in Mechanism and Machine Science, Guanajuato, Mexico, A11_512, available at: http: //www.dmg-lib.org/dmglib/handler?docum=22411009 (last access: 7 August 2018), 2011a.

Linß, S., Erbe, T., Theska, R., and Zentner, L.: The influence of asymmetric flexure hinges on the axis of rotation, 56th International Scientific Colloquium, Ilmenau, Germany, nbnresolving.de/urn:nbn:de:gbv:ilm1-2011iwk-006:6, $2011 \mathrm{~b}$.

Linß, S., Milojevic, A., Pavlovic, N. D., and Zentner, L.: Synthesis of Compliant Mechanisms based on GoalOriented Design Guidelines for Prismatic Flexure Hinges with Polynomial Contours, 14th World Congress 
in Mechanism and Machine Science, Taipei, Taiwan, https://doi.org/10.6567/IFToMM.14TH.WC.PS10.008, 2015.

Linß, S., Schorr, P., and Zentner, L.: General design equations for the rotational stiffness, maximal angular deflection and rotational precision of various notch flexure hinges, Mech. Sci., 8, 29-49, https://doi.org/10.5194/ms-8-29-2017, 2017a.

Linß, S., Schorr, P., Henning, S., and Zentner, L.: Contourindependent Design Equations For The Calculation Of The Rotational Properties Of Commonly Used And Polynomial Flexure Hinges, 59th Ilmenau Scientific Colloquium, Ilmenau, Germany, nbn-resolving.de/urn:nbn:de:gbv:ilm1-2017iwk-001:5, $2017 \mathrm{~b}$.

Linß, S., Gräser, P., Räder, T., Henning, S., Theska, R., and Zentner, L.: Influence of geometric scaling on the elasto-kinematic properties of flexure hinges and compliant mechanisms, Mech. Mach. Theory, 125, 220-239, https://doi.org/10.1016/j.mechmachtheory.2018.03.008, 2018.

Lobontiu, N.: Compliant Mechanisms: Design of Flexure Hinges, CRC Press, Boca Raton, Fla., 2003.

Lobontiu, N., Cullin, M., Ali, M., and Brock, J. M.: A generalized analytical compliance model for transversely symmetric three-segment flexure hinges, Rev. Sci. Instrum., 82, 105116, https://doi.org/10.1063/1.3656075, 2011.

Megaro, V., Zehnder, J., Bächer, M., Coros, S., Gross, M., and Thomaszewski, B.: A Computational Design Tool for Compliant Mechanisms, ACM Trans. Graph., 36, 1-12, https://doi.org/10.1145/3072959.3073636, 2017.

Meng, Q., Li, Y., and Xu, J.: New empirical stiffness equations for corner-filleted flexure hinges, Mech. Sci., 4, 345-356, https://doi.org/10.5194/ms-4-345-2013, 2013.

Palmieri, G., Palpacelli, M. C., and Callegari, M.: Study of a Fully Compliant U-Joint Designed for Minirobotics Applications, J. Mech. Des., 134, 111003-111004, https://doi.org/10.1063/1.3137074, 2012.

Paros, J. M. and Weisbord, L.: How to design flexure hinges, Machine design, 25, 151-156, 1965.

Pei, X. and Yu, J.: ADLIF: a new large-displacement beam-based flexure joint, Mech. Sci., 2, 183-188, https://doi.org/10.5194/ms2-183-2011, 2011.

Schotborgh, W. O., Kokkeler, F. G., Tragter, H., and van Houten, F. J. A. M.: Dimensionless design graphs for flexure elements and a comparison between three flexure elements, Precis. Eng., 29, 4147, https://doi.org/10.1016/j.precisioneng.2004.04.003, 2005.

Smith, S. T.: Flexures: elements of elastic mechanisms, Gordon \& Breach, Amsterdam, 2000.

Smith, S. T., Chetwynd, D. G., and Bowen, D. K.: Design and assessment of monolithic high precision translation mechanisms, J. Phys. E, 8, 977-983, https://doi.org/10.1088/00223735/20/8/005, 1987.

Smith, S. T., Badami, V. G., Dale, J. S., and Xu, Y.: Elliptical flexure hinges, Rev. Sci. Instrum., 68, 1474-1483, https://doi.org/10.1063/1.1147635, 1997.

Tian, Y., Shirinzadeh, B., Zhang, D., and Zhong, Y.: Three flexure hinges for compliant mechanism designs based on dimensionless graph analysis, Precis. Eng., 34, 92-101, https://doi.org/10.1016/j.precisioneng.2009.03.004, 2010.
Tseytlin, Y. M.: Notch flexure hinges: An effective theory, Rev. Sci. Instrum., 73, 3363-3368, https://doi.org/10.1063/1.1499761, 2002.

Turkkan, O. A. and Su, H.-J.: DAS-2D: A concept design tool for compliant mechanisms, Mech. Sci., 7, 135-148, https://doi.org/10.5194/ms-7-135-2016, 2016.

Vallance, R. R., Haghighian, B., and Marsh, E. R.: A unified geometric model for designing elastic pivots, Precis. Eng., 32, 278288, https://doi.org/10.1016/j.precisioneng.2007.10.001, 2008.

van Beek, A.: Calculator for a single notched hinge, available at: http://www.tribology-abc.com/calculators/c14_2.htm, last access: 23 July 2018.

Venanzi, S., Giesen, P., and Parenti-Castelli, V.: A novel technique for position analysis of planar compliant mechanisms, Mech. Mach. Theory, 40, 1224-1239, https://doi.org/10.1016/j.mechmachtheory.2005.01.009, 2005.

Vink, J.: Vink, System Design \& Analysis - Calculating flexure hinges, available at: https://www.vinksda.nl/ toolkit-mechanical-calculations/calculating-flexure-hinges, last access: 23 July 2018.

Wang, R., Zhou, X., and Zhu, Z.: Development of a novel sort of exponent-sine-shaped flexure hinges, Rev. Sci. Instrum., 84, 095008, https://doi.org/10.1063/1.4821940, 2013.

Wu, Y. and Zhou, Z.: Design calculations for flexure hinges, Rev. Sci. Instrum., 73, 3101, https://doi.org/10.1063/1.1494855, 2002.

Yong, Y. K., Lu, T.-F., and Handley, D. C.: Review of circular flexure hinge design equations and derivation of empirical formulations, Precis. Eng., 32, 63-70, https://doi.org/10.1016/j.precisioneng.2007.05.002, 2008.

Zelenika, S., Munteanu, M. G., and Bona, F. De: Optimized flexural hinge shapes for microsystems and highprecision applications, Mech. Mach. Theory, 44, 1826-1839, https://doi.org/10.1016/j.mechmachtheory.2009.03.007, 2009.

Zentner, L.: Nachgiebige Mechanismen, De Gruyter Oldenbourg, München, 2014.

Zentner, L., Griebel, S., Wystup, C., Hügl, S., Rau, T. S., and Majdani, O.: Synthesis process of a compliant fluidmechanical actuator for use as an adaptive electrode carrier for cochlear implants, Mech. Mach. Theory, 112, 155-171, https://doi.org/10.1016/j.mechmachtheory.2017.02.001, 2017.

Zettl, B., Szyszkowski, W., and Zhang, W. J.: On Systematic Errors of Two-Dimensional Finite Element Modeling of Right Circular Planar Flexure Hinges, J. Mech. Des., 127, 782-787, https://doi.org/10.1115/1.1898341, 2005.

Zhu, B. L., Zhang, X. M., and Fatikow, S.: Design of single-axis flexure hinges using continuum topology optimization method, Sci. China Technol. Sci., 57, 560-567, https://doi.org/10.1007/s11431-013-5446-4, 2014. 